\title{
Characterisation of the fabrication process of freestanding SU-8 microstructures integrated in printing circuit board in microelectromechanical systems
}

ARTICLE in MICRO \& NANO LETTERS · MARCH 2010

Impact Factor: 0.8· DOI: 10.1049/mnl.2009.0097 · Source: IEEE Xplore

CITATIONS

17

4 AUTHORS, INCLUDING:

Francisco Perdigones

Universidad de Sevilla

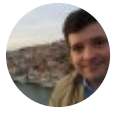

Antonio Luque

Universidad de Sevilla

38 PUBLICATIONS 111 CITATIONS

42 PUBLICATIONS 183 CITATIONS

SEE PROFILE

SEE PROFILE

José M. Quero

Universidad de Sevilla

145 PUBLICATIONS 615 CITATIONS

SEE PROFILE 


\title{
CHARACTERIZATION OF THE FABRICATION PROCESS OF FREE-STANDING SU-8 MICROSTRUCTURES INTEGRATED IN PCBMEMS
}

\author{
F. Perdigones, J. M. Moreno, A. Luque and J.M. Quero \\ Departamento de Ingeniería Electrónica, Escuela Superior de Ingenieros, Universidad de
} Sevilla, Avenida de los Descubrimientos S/N.

Email: perdi@gte.esi.us.es, jmmoreno@gte.esi.us.es

\begin{abstract}
The characterization of the fabrication process to develop free-standing SU-8 structures integrated in PCBMEMS (Printing Circuit Board in Microelectromechanical Systems) technology is presented. SU-8 microcantilevers, microbridges, microchannels and micromembranes have been fabricated following the described procedure. Adherence between FR4 substrate and SU-8 has been studied using the destructive blister method, determining the surface energy. Residual thermal stress has also been analyzed for this integration and compared when using other substrates. Moreover, a study of the copper wet etching with cupric chloride has been performed in order to characterize how this isotropic etching affects the geometry of the copper structures. Finally, stiction has been observed and examined, determining the adhesion energy responsible of this effect.
\end{abstract}

\section{INTRODUCTION}

During the last decade, the fabrication of microstructures employing alternative technologies to silicon and innovative processes has rapidly increased in the field of microelectromechanical systems (MEMS). In this respect, polymer technology has made possible to overcome most of the high costs and restrictions related to traditional silicon manufacturing processes.

Specifically, SU-8 is an epoxy-based negative photoresist which can be patterned using standard mask photolithography, offering a simple alternative for rapid prototyping [1]. Another technology that is becoming important in microfabrication area is PCBMEMS, which involves 
electronic, mechanical and fluidic components by adding some new steps in common PCB fabrication [2]. The main advantage lies in its low cost, robustness and high integration, making the fabrication of complex microdevices possible by the combination of a few PCB layers and simple processes.

By the integration of both SU-8 and PCBMEMS technologies, it is possible to develop many structures in a wide range of applications, without using expensive clean room facilities or special equipment. Several authors have proposed different contributions to this area, coating SU-8 over PCBs for encapsulating microchips [3], as planarization layers [4] and also for microchannel manufacturing [5]. Another remarkable application is the use of conductive SU-8 [6] or ferromagnetic SU-8 [7], making possible an electronic integration of SU-8 and PCBMEMS.

Leïchlé et al [8] reported a resonant micromachined compass using the PCB copper as a sacrificial layer to fabricate a free-standing SU-8 structure, providing new possibilities towards the simple and rapid processing of micromechanical components. Although thermally decomposable polymers, metal layers ( $\mathrm{Au}, \mathrm{Ti}$ or $\mathrm{Cr}$ ) [9] or positive-tone-photoresist [10] have been mainly chosen as sacrificial materials, the copper from a commercially standard PCB presents some advantages when compared with those alternatives.

Copper can be directly patterned in the substrate by photolithography so deposition steps are not required. Furthermore, SU-8 processing temperatures or the solvents required for developing have no effect on the copper properties and vice versa: the SU-8 keeps its mechanical properties unaffected during the wet etching step when removing the sacrificial copper layer. This fabrication process allows the rapid and inexpensive manufacturing of freestanding microstructures in a large scale of integration by means of a unique SU-8 deposition, without using expensive facilities such as laser or RIE systems.

In this paper, the characterization of the integration of free-standing SU-8 microstructures in PCBMEMS is analyzed and presented. Adherence between SU-8 and PCB substrate, residual thermal stress, stiction and copper wet etching effects on SU-8 are studied in order to achieve a 
successful integration of both technologies. Microcantilevers, microbridges, microchannels and other structures have successfully been fabricated following the described procedure.

\section{FABRICATION PROCESS}

In this section, a microcantilever process flow is detailed to illustrate a simple structure fabrication by applying the technology characterized in this paper. The fabrication process is easy to be carried out in a few steps and integrates inexpensive materials like PCB and SU-8, as is shown in Fig. 1.

Following the PCBMEMS technology, a conventional single-sided copper-plated rigid material, flame retardant 4 (FR4) is used as a substrate. The thickness of the FR4 and the copper layer are selectable $(18-105 \mu \mathrm{m}$ for the copper and 100-1500 $\mu \mathrm{m}$ for the FR4), choosing commercially standard thicknesses of $70 \mu \mathrm{m}$ and $1500 \mu \mathrm{m}$, respectively. The copper is coated with a few micrometers positive photoresist layer, making its photolithographic process possible.

The fabrication process starts when photolithography is applied on the top side of the PCB, Fig. 1(a), in order to determine the sacrificial areas where the cantilever will be released and the areas where it will be anchored to the FR4 substrate. After UV light exposure with mask-1 for $2.5 \mathrm{~min}$, the exposed areas are etched using cupric chloride. The remaining photoresist is then stripped with acetone and the PCB air-dried, Fig. 1(b).

The next step is the deposition of an SU-8 layer over the PCB, Fig. 1(c), using a spin coater SMA AC 6000 (supplied by SMA Inc.). To obtain thin SU-8 depositions, negative photoresist SU-8 2025 (Microchem) is used, achieving a SU-8 layer with a thickness of $100 \mu \mathrm{m}$. In the PCB areas where sacrificial copper is under the deposited SU-8, the thickness of the resist layer is $30 \mu \mathrm{m}$ from copper. Deposition details are studied and discussed in detail in section III.

In the next step, the SU-8 is soft baked at $65{ }^{\circ} \mathrm{C}$ for $5 \min$ and at $95{ }^{\circ} \mathrm{C}$ for $60 \mathrm{~min}$ on a hotplate. When the resist is cured and cooled down at room temperature for $10 \mathrm{~min}$, a mask with the cantilever pattern is used for lithography (mask-2). This mask must be aligned with the sacrificial copper area in order to obtain a cantilever with one end anchored to the substrate. 
SU-8 is then exposed to UV light with mask-2 for 2 min, Fig. 1(d). Then, a post exposure bake step (PEB) is performed at $65^{\circ} \mathrm{C}$ for $5 \mathrm{~min}$ and $95^{\circ} \mathrm{C}$ for $10 \mathrm{~min}$ on a hotplate in order to crosslink the resist. After $10 \mathrm{~min}$ at room temperature the following stage is to immerse the board in the Mr600 developer (Microchem) for $5 \mathrm{~min}$. Then the patterned SU-8 is rinsed in isopropyl alcohol (IPA).

In Fig. 1 (e) the resulting SU-8 structure after development can be seen. It is important to highlight that the SU-8 layer is flat over the whole board. The SU-8 is in contact with the substrate in the left side of the copper, whereas the rest of the cantilever is separated $70 \mu \mathrm{m}$ over the substrate.

Finally, in step (f) sacrificial copper is etched using cupric chloride, being removed from beneath the SU-8 and leaving the structure released. No SU-8 degradation is observed thorough the complete etching process. After this, the board is immersed in DI water to remove the etchant completely. Copper wet etching steps will be discussed in depth in section VI.

SU-8 microcantilevers are illustrated in Fig. 2 before and after the copper sacrificial layer etching step. The cantilevers present a length of $400 \mu \mathrm{m}$, a width of $50 \mu \mathrm{m}$ and a thickness of 30 $\mu \mathrm{m}$. The thickness of the copper layer $(70 \mu \mathrm{m})$ provides enough distance between the cantilevers and the FR4 substrate, which could be easily adjusted by selecting a PCB with a thicker or thinner copper layer.

In order to confirm the potential of this technology, several structures were fabricated following the described fabrication process, as is shown in Fig. 3. The thickness of the SU-8 free-standing layer for all of them was $30 \mu \mathrm{m}$. In Fig. 3(a), a microbridge with a length of 500 $\mu \mathrm{m}$ and a width of $100 \mu \mathrm{m}$ is illustrated after copper removal from beneath. A free-standing double-clamped serpentine spring with a minimum cross-section of $50 \mu \mathrm{m} \times 30 \mu \mathrm{m}$ is also illustrated in Fig. 3(b), with a length of $2 \mathrm{~mm}$ and a width of $800 \mu \mathrm{m}$.

In Fig. 3(c), a free-standing membrane is shown with a radius of $250 \mu \mathrm{m}$. The external radius where the structure was held by means of four suspensions was $450 \mu \mathrm{m}$. The suspensions presented a width of $50 \mu \mathrm{m}$. Finally, a circular geometry microstructure with concentric SU-8 
rings is shown in Fig. 3(d). Four cylindrical columns with diameters of $200 \mu \mathrm{m}$ hold the structure. The rings presented a width of $100 \mu \mathrm{m}$, with an external ring radius of $800 \mu \mathrm{m}$.

\section{SU-8 DEPOSITION ON FR4}

The first issue to consider is the SU-8 2025 deposition on the FR4 substrate. The thickness of this layer depends on the spinning speed, the time being spun and the SU-8 viscosity. Experimental tests were performed at different spinning speeds for 60 seconds and the results compared with the SU-8 deposition thicknesses on silicon substrate (Microchem), as is shown in table 1.

Table 1. SU-8 2025 layer thickness over FR4 and silicon as a function of the spin speed. The reported thickness presented a deviation of $5 \%$.

\begin{tabular}{|c|c|c|c|c|}
\hline R.P.M. & $\mathbf{7 0 0}$ & $\mathbf{1 0 0 0}$ & $\mathbf{1 5 0 0}$ & $\mathbf{2 5 0 0}$ \\
\hline Thickness over FR4 $(\boldsymbol{\mu m})$ & 100 & 75 & 50 & 25 \\
\hline Thickness over Si $(\boldsymbol{\mu m})$ & 100 & 80 & 60 & 35 \\
\hline
\end{tabular}

According to these results, the SU-8 deposition thickness over FR4 and silicon substrate are similar. Following the microcantilever fabrication process reported in section II, the spin speed selected to achieve a SU-8 layer on FR4 with a thickness of $100 \mu \mathrm{m}$ was $700 \mathrm{rpm}$ for 60 s.

Another issue is to achieve a reasonable flatness of the deposition when the copper has been patterned and the board surface is not completely flat. This problem can be overcome if the design thickness of the SU-8 layer is sufficiently larger than the thickness of the sacrificial copper, and when the substrate area is large enough compared with sacrificial copper area.

\section{ADHERENCE}

In this section the adhesion between SU-8 and FR4 is discussed. Low values for the surface energy between both materials could cause delaminations, making impossible a correct device fabrication. To determine the surface energy, $\gamma_{1}$, a destructive blister test [11] is 
performed with the set up illustrated in Fig. 4. The specimen used for this test consists on a drilled FR4 with a thin SU-8 2025 layer spin coated over it.

When a pressure is applied through the FR4, the SU-8 membrane over it will deform under the exerted pressure while the rest of the membrane is fixed to the substrate. When a critical pressure $P$ is reached, a crack will propagate through the bond interface, assuming the bond surface energy weaker than the FR4 fracture energy. The crack travels through the interface until the membrane bursts under the exerted pressure, which has the same value than the critical pressure due to the crack fast propagation.

From the burst pressure $P$, the surface energy of the bond between SU-8 and FR 4 can be determined by the expression:

$$
\gamma_{1}=\frac{3 a^{4}\left(1-v^{2}\right)}{32 \pi t^{3} E} P^{2}
$$

Where $a$ is the blister radius, $t$ is the membrane thickness, $P$ is the critical pressure, $v$ is the SU-8 Poisson coefficient and $E$ its Young modulus.

Several tests were performed with a SU-8 deposition thickness $t=80 \mu \mathrm{m}$ and a fixed radius $a=1250 \mu \mathrm{m}$ for the FR4 inlet hole. A compressed air supply with a pressure transducer is connected to the hole, and the inlet pressure increased until a failure of the bond interface is achieved with the corresponding membrane crack. Table 2 summarizes the surface energy between SU-8 and FR4 calculated with the expression (1) for each inlet pressure required to crack during experimental tests.

Table 2. Surface energy between SU-8 and FR4 resulting from blister tests.

\begin{tabular}{|c|c|c|c|c|c|}
\hline Test \# & $\mathbf{1}$ & $\mathbf{2}$ & $\mathbf{3}$ & $\mathbf{4}$ & $\mathbf{5}$ \\
\hline $\mathbf{P}(\mathrm{MPa})$ & 0.200 & 0.205 & 0.225 & 0.210 & 0.200 \\
\hline$\gamma\left(\mathrm{J} / \mathrm{m}^{2}\right)$ & 1.231 & 1.293 & 1.558 & 1.357 & 1.231 \\
\hline & \multicolumn{2}{|c}{$\boldsymbol{\mu}_{\gamma}=1.334 \mathrm{~J} / \mathrm{m}^{2}$} & \multicolumn{3}{c|}{$\boldsymbol{\sigma}_{\gamma}=0.136 \mathrm{~J} / \mathrm{m}^{2}$} \\
\cline { 2 - 6 }
\end{tabular}

These experimental tests provided an average surface energy $\mu_{\gamma}$ of $1.334 \mathrm{~J} / \mathrm{m}^{2}$ with a standard deviation $\sigma_{\gamma}$ of $0.136 \mathrm{~J} / \mathrm{m}^{2}$, according to the described fabrication conditions. This value is larger than the surface energy of SU-8-to-PDMS [12] and the $\mathrm{SiO}_{2}$-SU-8-pyrex bonded 
wafers [13], $0.047 \mathrm{~J} / \mathrm{m}^{2}$ and $0.5 \mathrm{~J} / \mathrm{m}^{2}$ respectively, and comparable to Silicon-to-Pyrex glass bonding surface energy, $1.3 \mathrm{~J} / \mathrm{m}^{2}[14]$.

\section{RESIDUAL THERMAL STRESS}

The residual stress during fabrication must also be evaluated. This stress appears due to the different values of the coefficient of thermal expansion (CTE) between SU-8 and the used substrate. The expression to determine this value is [15]:

$$
\sigma_{t h}=\left(\alpha_{S U-8}-\alpha_{F R 4}\right) \frac{E_{S U-8}}{1-v_{S U-8}}\left(T_{P E B}-T_{0}\right)
$$

Where $\alpha$ is the CTE of the corresponding material, $v$ and $E$ are the SU-8 Poisson ratio and Young modulus respectively, $T_{P E B}$ is the PEB temperature and $T_{0}$ is the room temperature.

For a given $T_{P E B}$, as the difference for the CTE values is higher, the residual stress between both materials increases. With a CTE about $13 \mathrm{ppm} / \mathrm{K}$ for the FR4 and $52 \mathrm{ppm} / \mathrm{K}$ for the SU-8, the residual stress between both materials results in $13.4 \mathrm{MPa}$ under fabrication conditions. Therefore, the residual thermal stress is lower than the corresponding value for silicon under the same PEB temperature (CTE of $3 \mathrm{ppm} / \mathrm{K}$ ), or pyrex (CTE of $3.2 \mathrm{ppm} / \mathrm{K}$ ). However, the residual stress can be controlled by reducing the PEB temperature as is shown in (2), but the baking times must be increased.

\section{COOPER WET ETCHING}

Another issue in the fabrication process is the use of cupric chloride to remove the copper during patterning wet etching and sacrificial wet etching steps. The cupric chloride attacks the copper isotropically, so the geometry of the copper areas after the patterning etching procedure will be affected.

To illustrate this issue, microchannels were fabricated by burying copper lines with SU-8 and leaving a side open to allow the etchant to remove the sacrificial copper. The copper line cross-section was $200 \mu \mathrm{m} \times 70 \mu \mathrm{m}$, however the shape achieved after patterning wet etching 
step is illustrated in Fig. 5(a). This photograph clearly shows the isotropic etching effect on both edges of the copper line, changing its cross-section. This result must be taken into account, because the cross-section of the microchannel after sacrificial etching will be determined by the cross-section of the copper line previously patterned, as is shown in Fig. 5(b).

The time required to etch a given length of the sacrificial copper to create the microchannel was measured and illustrated in Fig. 6. This curve is also compared with a similar microchannel fabricated in a PCB with a $35 \mu \mathrm{m}$ copper line (cross-section of $200 \times 35 \mu \mathrm{m}$ ), in order to determine the fastest copper etching rate. The length of the etched copper was shorter for the microchannel where a smaller section made the etching action more difficult, resulting in a higher etching rate for the $70 \mu \mathrm{m}$ copper line.

The etching time for the free-standing fabricated structures reported in section II depended on the holes and cavities patterned on the SU-8 structure over the copper layer, which allow the contact between the sacrificial copper and the etchant through the SU-8. However, the etching time reported was always less than 25 minutes, with a maximum copper etching rate of $3 \mu \mathrm{m} / \mathrm{min}$

The presence of cupric chloride during sacrificial wet etching step did not cause damage in the SU-8 structure or changed the SU-8 mechanical properties during the experiments.

\section{STICTION}

A typical problem of the wet etching process is the stiction, which causes that fabricated structures stick on the substrate from a critical length due to the smoothness of the surface [16]. In this section, the stiction effect between the free-standing SU-8 structures and the FR4 substrate is studied and the adhesion energy determined. The process to obtain this value consists on fabricating several cantilevers with different lengths so that the critical length is evaluated, and the adhesion energy, $\gamma_{2}$ is obtained using the expression (3):

$$
\gamma_{2}=\frac{3 E t^{3} g^{2}}{8 l_{c r i t}^{4}}
$$


Where $E$ is the SU-8 Young modulus, $t$ is the thickness of the cantilever, $g$ is the gap between cantilever and substrate, and $l_{\text {crit }}$ is the critical length when the stiction appears. This phenomenon is observed for cantilevers with lengths from $1900 \mu \mathrm{m}$ and thicknesses of $30 \mu \mathrm{m}$, where the determined adhesion energy is $16.75 \pm 0.35 \mathrm{~mJ} / \mathrm{m}^{2}$. This value is lower than the minimum one reported for silicon substrate with $\mathrm{H}_{2} \mathrm{O}$ as liquid, $50 \mathrm{~mJ} / \mathrm{m}^{2}$ [17].

\section{CONCLUSION}

The characterization of the fabrication process which integrates free-standing SU-8 structures in PCBMEMS technology has been presented. SU-8 microstructures were fabricated following the manufacturing steps in order to analyze the different aspects involved in the process. Deposition details and adherence between SU-8 and FR4 substrate, residual thermal stress, copper wet etching effects on SU-8 and stiction are studied to achieve a successful integration of both technologies.

A blister test was carried out to determine the surface energy between SU-8 and FR4, resulting a high value of $1.334 \mathrm{~J} / \mathrm{m}^{2}$. A theoretical study of thermal stress was also performed, providing a low residual stress about 13.4 MPa. Cupric chloride did not damage the SU-8 during sacrificial etching step, however the previous patterning wet etching isotropically changed the copper geometry. Stiction was also studied in a group of cantilevers, resulting an adhesion energy of $16.75 \mathrm{~mJ} / \mathrm{m}^{2}$. With this value, the free-standing structures critical length with different gaps and thicknesses can be estimated.

These characterized parameters have demonstrated that the integration of SU-8 and PCBMEMS technologies allows the simple and rapid fabrication of robust and inexpensive microdevices. Finally, the presented work could serve as a first step towards the fabrication of SU-8/PCB hydrid devices.

\section{REFERENCES}


[1] H. Lorenz, M. Despont, N. Fahrni, N. LaBianca, P. Renaud, and P. Vettiger, "SU-8: a lowcost negative resist for MEMS", Journal of Micromechanics and Microengineering, Volume 7 Issue 3, 1997 Pages $121-124$

[2] T. Merkel, M. Graeber, L. Pagel, “A new technology for fluidic microsystems based on PCB technology", Sensors and Actuators, Volume 77, 1999 Pages 98-105

[3] P.A. Hammond, D.R.S. Cumming, "Encapsulation of a liquid-sensing microchip using SU-8 photoresist", Microelectronic Engineering, Volume 73-74, 2004 Pages 893-897

[4] A. Petropoulos, D. Goustouridis, T. Speliotes, G. Kaltsas, "Demonstration of a new technology which allows direct sensor integration on flexible substrates", Eur. Phys. J. Appl. Phys., Volume 46 No 1, 2009 (64p.)

[5] K. Kontakis, A. Petropoulos, G. Kaltsas, T. Speliotis, E. Gogolides, "A novel microfluidic integration technology for PCB-based devices: Application to microflow sensing", Microelectronic Engineering, Volume 86, 2009 Pages 1382-1384.

[6] S. Jiguet, A. Bertsch , H. Hofmann , P. Renaud, "Conductive SU8 Photoresist for Microfabrication," Advanced Functional Materials, Volume 15 Issue 9 (2005) pages 1511-1516. [7] N. Damean, B. A. Parviz, J. Ng Lee, T. Odom and G. M. Whitesides, "Composite ferromagnetic photoresist for the fabrication of microelectromechanical systems," J. Micromech. Microeng. Volume 15, (2005) pages 29-34.

[8] T. C. Leïchlé, M. Von Arx, S. Reiman, I. Zana, W. Ye and M. G. Allen. "A low-power resonant micromachined compass," J. Micromech. Microeng. Volume 14 (2004) Pages 462-470 [9] P.J. Joseph, H. A Kelleher, S. A. Bidstrup-Allen, P. A. Kohl, "Improved fabrication of micro air-channels by incorporation of a structural barrier", J. Micromech. Microeng., Volume 15, 2005 Pages 35-42

[10] A. Ezkerra, L. J. Fernández, K. Mayora, J. M. Ruano-López, "Fabrication of SU-8 freestanding structures embedded in microchannels for microfluidic control", J. Micromech. Microeng., Volume 17, 2007 Pages 2264-227. 
[11] A. Doll, M. Rabold, F. Goldschmidtböing and P. Woias, "Versatile low temperature wafer bonding and bond strength measurement by a blister test method," Microsyst Technol (2006) Volume 12 Pages 418-429.

[12] M J Kastantin, S Li, A P Gadre, L Q Wu, W E Bentley, G F Payne, G W Rubloff and R Ghodssi, "Integrated fabrication of polymeric devices for biological applications," Sensors Materials Volume 15 (2003) pages 295-311

[13] Li S, Freidhoff C B, Young R M and Ghodssi R "Fabrication of micronozzles using lowtemperature wafer-level bonding with SU-8," J. Micromech. Microeng. Volume 13 (2003) pages $732-738$

[14] Alexander Doll, Martin Rabold Frank Goldschmidtböing and Peter Woias," Versatile low temperature wafer bonding and bond strength measurement by a blister test method," Microsyst Technol Volume 12 (2006) pages 418-429

[15] S. Keller, G. Blagoi, M. Lillemose, D. Haefliger, A. Boisen, "Processing of thin SU-8 films," Journal of Micromechanics and Microengineering Volume 18, Number 12 (2008) 125020

[16] Niels Tas, Tonny Sonnenberg, Henri Jansen, Rob Legtenberg and Miko Elwenspoek, "Stiction in surface micromachining," J. Micromech. Microeng. Volume 6 (1996) pages 385397

[17] R. Legtenberg, H.C.A. Tilmans, J. Elders and M. Elwenspoek "Stiction on surface micromachined structures after rising and drying: model and investigation of adhesion mechanisms", Sensors and Actuators, Volume 43, 1994 Pages 230-238 